\title{
Cascaded Mach-Zehnder architectures for photonic integrated delay lines
}

\author{
Abi Waqas, Daniele Melati, and Andrea Melloni
}

\begin{abstract}
A continuously tunable integrated optical delay line architecture with large bandwidth and bandwidth-delay product is proposed and demonstrated. The non-resonant delay line is based on cascaded Mach-Zehnder interferometers with tunable couplers. The device is operated with a single control signal and outperform the common ring-resonator-based delay lines in terms of bandwidth-delay product. A device with a delay tuning range of $124 \mathrm{ps}$ is experimentally demonstrated over a transmission bandwidth of $4.5 \mathrm{GHz}$.
\end{abstract}

Index Terms-Optical delay lines, optical filter, integrated optics devices, Mach-Zehnder interferometer, true-time delay.

\section{INTRODUCTION}

$\mathbf{R}$ EALIZING a continuously tunable, integrated delay line without mechanical moving parts is not an easy task in many fields including optics, electronics, microwave, acoustic and so on. In photonics the attractive possibility of on-chip integration with other optical functionalities led in the last two decades to a collection of devices which can be used for synchronization, buffering, multiplexing and true-time delay in a plethora of applications. Ring resonators and photonic crystals are the most common elements to conceive controllable integrated circuits able to tune continuously the group delay up to several hundreds of picoseconds and even few nanoseconds [1]-[9]. Both ring resonators and photonics crystals exploit their resonant (edge) spectral behavior to achieve compact footprint devices at the expense of narrow bandwidth [10]. Moreover, any additional imperfection such as lumped losses in directional couplers or roughness induced scattering is coherently enhanced by the resonant nature, degrading the insertion loss and spectral purity of the delay line.

It should be noticed that whatever it is the circuit architecture, the delay $\tau$ is proportional to the group optical length traveled by the light. The propagation insertion loss is therefore $\mathrm{IL}=\alpha \tau c / n_{g}$ where $\mathrm{c}$ is the speed of light, and $\alpha$ and $n_{g}$ are the waveguide attenuation and group refractive index, respectively. The ultimate insertion loss per unit delay depends only on the ratio $\alpha / n_{g}$, that is on the waveguide characteristics and not on the circuit topology. The waveguide with a lower $\alpha / n_{g}$ ratio is hence better suited for delay line applications.

Other than losses, the most unfavorable aspect of a resonance-based delay lines is the limit in the bandwidth-delay

A. Waqas and A. Melloni are with the Dipartimento di Elettronica, Informazione e Bioingegneria, Politecnico di Milano, via Ponzio 34/5, 20133 Milano, Italy. D. Melati is with National Research Council Canada, 1200 Montreal Rd., Ottawa, Ontario K1A 0R6, Canada. A. Waqas is also with the Departement of Telecommunication Engineering, Mehran University of Engineering and Technology, Jamshoro, 76062 Sindh, Pakistan.

Manuscript received XXXXX XX, XXXX; revised XXXXXX XX, XXXX. product since a single delay element induces a delay that is a fraction of the inverse of its bandwidth. Coupled resonators can increase the operational bandwidth but generally require complex tuning and control strategies. On the contrary, nonresonant delay lines achieve a much larger bandwidth and an easier control of the tuning. The most common non-resonant delay lines are realized using $2 \times 2$ switches to select paths of different physical lengths thus changing the total propagation length and delay but providing discrete steps instead of a continuous tunable delay [2], [11], [12].

Recently, we have proposed a non-resonant Mach-Zehnderbased integrated optical delay line that is continuously tunable with a large bandwidth-delay product and a very low chromatic dispersion [13]. Two tunable couplers allow the full tunability of the dealy. In this letter, we propose an architecture implementing a two-stage Mach-Zehnder-based delay line (2MZDL) with improved performance. We demonstrate that with the proper control scheme the novel architecture doubles the group delay tunability range compared to a single-stage MachZehnder delay line (1MZDL) and achieves a bandwidth-delay product larger by a factor 2 and 5 compared to a $1 \mathrm{MZDL}$ and a ring resonator, respectively. Both single- and two-stage structures have a simple, robust and hitless control scheme since the delay can be adjusted without affecting power transmission at the operative frequency during operation. The single-stage delay line is reviewed and the two-stage device is proposed, analyzed and compared also with a classical ring delay line. Finally, the experimental spectral characterization of a 1MZDL, fabricated in indium phosphide (InP) technology, is reported, demonstrating a continuously tunable delay from zero to 124 ps over a minimum $3 \mathrm{~dB}$ transmission bandwidth of $4.5 \mathrm{GHz}$.

\section{MACH-ZEHnder-BASED DELAY Lines}

The schematic of the proposed optical delay line is shown in Fig. 1. It includes two cascaded Mach-Zehnder interferometers and three tunable couplers. All tunable couplers $\left(\mathrm{K}_{1}, \mathrm{~K}_{2}\right.$ and $\mathrm{K}_{3}$ ) are realized using balanced Mach-Zehnder interferometer equipped with thermal phase shifters on one arm to vary the coupling ratios continuously from 0 to 1 . Both Mach-Zehnder stages have the same unbalance length $\Delta \mathrm{L}$ and a phase shifter on one arm to tune the operative frequency of the device to match that of the incoming signal.

The 1MZDL includes only the first two tunable couplers $K_{1}$ and $K_{2}$ (in dashed box of Fig. 1) and the first Mach-Zehnder. The maximum achievable group delay $T$ depends on $\Delta \mathrm{L}$ as $T=\left(n_{g} \Delta L\right) / c=1 / \mathrm{FSR}$ where $c$ is the speed of light and 


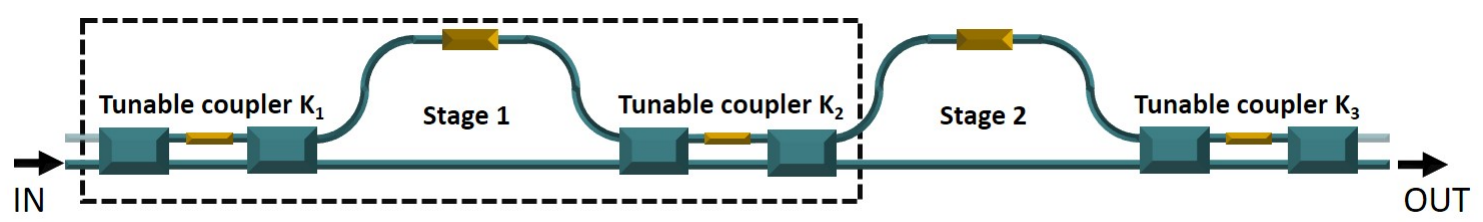

Fig. 1. Schematic of the proposed integrated optical delay line based on cascaded Mach-Zehnder interferometers with three tunable couplers. The box with dashed lines includes the schematic of the single stage delay line.

FSR is the Free Spectral Range. The device has to be used in bar state only, with input and output ports shown in Fig. 1. The two coupling ratios are chosen such that $K_{1}=K_{2}=K$.

If $H(\omega)$ is the transfer function of the 1MZDL in bar state and $\tau=\partial(\angle H) / \partial \omega$ the corresponding group delay (with $\angle H$ the phase of $H$ ), the normalized group delay can be written as [13]

$$
\tilde{\tau}=\frac{K(K-\cos \Delta \varphi+K \cos \Delta \varphi)}{(1-K)^{2}-2 K(1-K) \cos \Delta \varphi+K^{2}},
$$

where $\tilde{\tau}=\left(\tau-\tau_{0}\right) / T, \tau_{0}$ is the minimum delay, associated to the shortest branch of the interferometer, and $\Delta \varphi(\omega)$ the phase difference induced by the unbalance. The simulated intensity and normalized group delay of the transfer function $H$ are shown in Fig. 2(a) and (b), respectively, as function of the frequency for few values of K. $\omega_{0}$ is the operative central frequency for which $\Delta \varphi\left(\omega_{0}\right)$ is an odd multiple of $\pi$.

For $\mathrm{K}=0$ and $\mathrm{K}=1$ all the input light is coupled to shortest $(\tilde{\tau}=0)$ and longest $(\tilde{\tau}=1)$ arm, respectively, and then directed to the output. In these cases both the transmission and the group delay are frequency independent. At $\mathrm{K}=0.5$ the classical transfer function of the Mach-Zehnder is formed. Interestingly, the group delay characteristic is once again frequency independent and $\tilde{\tau}=0.5$ (except for $\omega=\omega_{0} \pm F S R / 2$ where the delay is undefined). For all the other values of $\mathrm{K}$ both the intensity and the group delay are frequency dependent but at the operating frequency $\omega_{0}$ (marked with a vertical dashed line in Fig. 2) the transmission does not depend on $\mathrm{K}$ whereas the normalized group delay increases linearly with $\mathrm{K}$. The transmission bandwidth of the 1MZDL around $\omega_{0}$ is $\mathrm{B}=(\mathrm{FSR} / \pi) \cos ^{-1}\left[(K-0.5)^{2} / K(1-K)\right]$. The minimum bandwidth is equal to FSR $/ 2$ and is obtained for $\mathrm{K}=0.5$ where the group delay is $\tau\left(\omega_{0}\right)=T / 2$.

The 2MZDL consists in two cascaded identical MachZehnder and three tunable couplers with coupling ratio equal to

$$
\begin{aligned}
& K_{1}=K_{3}=K=\sin ^{2}(\theta) \\
& K_{2}=\sin ^{2}(2 \theta)
\end{aligned}
$$

where $\theta$ is the phase shift induced by the coupler controller. With this control scheme the normalized group delay of the 2MZDL is given by

$\tilde{\tau}=\frac{2 K\left[(\cos \Delta \varphi+1)\left(4 K^{2}-6 K\right)+(2 \cos \Delta \varphi+3)\right]-1}{4 K(K-1)(2 K-1)^{2}(\cos \Delta \varphi+1)^{2}+1}+1$

At the operative central frequency $\omega_{0}, \tilde{\tau}$ varies linearly from 0 to 2 as $\mathrm{K}$ varies from 0 to 1 . The simulated spectral intensity transmission and normalized group delay are shown in Fig.

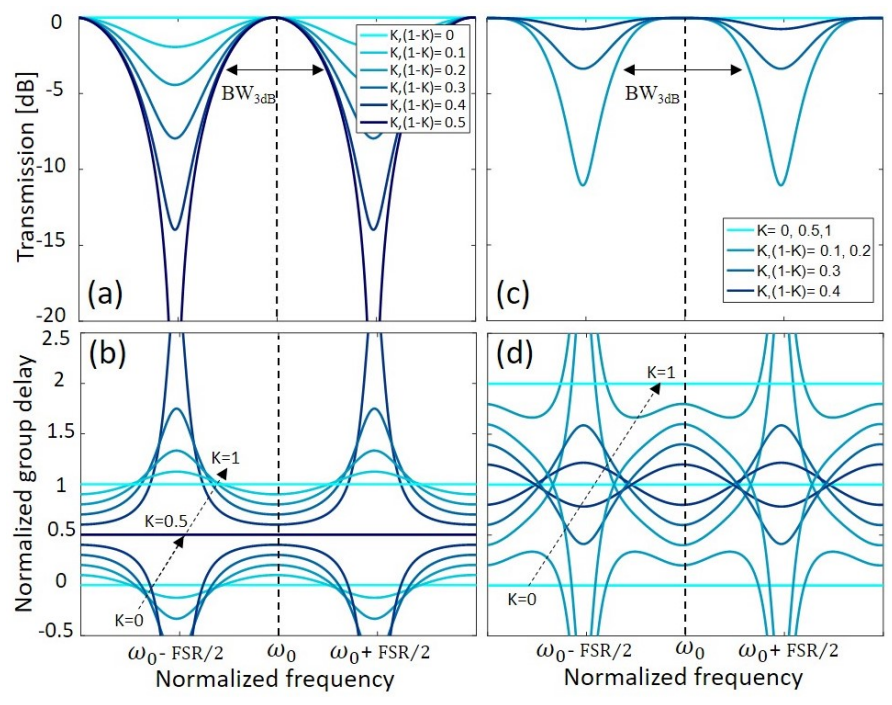

Fig. 2. Simulated transmission and normalized group delay of a 1MZDL $(\mathrm{a}, \mathrm{b})$ and a 2MZDL $(\mathrm{c}, \mathrm{d})$ as function of the normalized frequency for different values of $\mathrm{K}$. Vertical dashed lines show the operative frequency.

2(c) and (d), respectively, for the entire range of $\mathrm{K}$ values. The closed form expression of the 2MZDL $3 \mathrm{~dB}$ transmission bandwidth is not reported here for brevity but, interestingly, as reported in the next section the bandwidth is about $27 \%$ larger than the single-stage structure while the induced delay is twice that of the 1MZDL.

\section{Delay Line Characteristics}
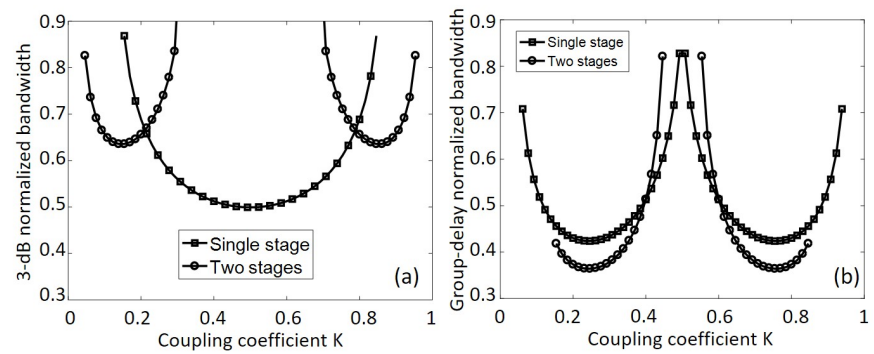

Fig. 3. (a) Normalized $3 \mathrm{~dB}$ transmission bandwidth and (b) group-delay bandwidth as function of the coupling coefficient $K$ for both 1MZDL and 2MZDL. The $3 \mathrm{~dB}$ bandwidth is defined on transmission spectrum while the group-delay bandwidth is defined where the delay is changed by $10 \%$ around the operative frequency $\omega_{0}$.

Figure 3(a) shows the $3 \mathrm{~dB}$ transmission bandwidth around the operative frequency $\omega_{0}$ normalized to the FSR of both 1MZDL and 2MZDL as a function of the coupling coefficient 
$\mathrm{K}$. When the depth of the notches in the spectral response reduces below $3 \mathrm{~dB}$ the bandwidth is not defined and hence not shown. For both delay lines the bandwidth can be considered infinite around $\mathrm{K}=0$ and $\mathrm{K}=1$. Further, the bandwidth of the $2 \mathrm{MZDL}$ is practically infinite in the range $\mathrm{K}=0.3$ to 0.7 . The minimum 3-dB bandwidth occurs for $\mathrm{K}$ equals to 0.5 for the $1 \mathrm{MZDL}$ and for $\mathrm{K}$ equal to 0.14 and 0.86 for the $2 \mathrm{MZDL}$, the latter being about $27 \%$ larger. It is also interesting to note the behavior of the two delay lines in terms of group-delay bandwidth, defined as the range around the operative frequency $\omega_{0}$ where the normalized delay varies by $10 \%$, as shown in Fig. 3(b). Also in this case the reported group-delay bandwidth is normalized to the FSR. For both 1MZDL and 2MZDL the group-delay bandwidth reaches its minimum around $\mathrm{K}=0.25$ and $\mathrm{K}=0.7$ and it is infinite for $\mathrm{K}=0, \mathrm{~K}=0.5$ and $\mathrm{K}=$ 1, consistently with Fig. 2 (b) and (d). Since the 2MZDL has a larger dispersion compared to the $1 \mathrm{MZDL}$, its group-delay bandwidth is smaller of about $16 \%$ around the minima.

The dependence of normalized group delay on the coupling coefficient $\mathrm{K}$ at the operative frequency for both 1MZDL and 2MZDL is shown in Fig. 4(a), right-hand side axis. As already discussed, normalized group delay linearly changes with $\mathrm{K}$ from 0 to 1 (squares) for $1 \mathrm{MZDL}$ and from 0 to 2 (circles) for 2MZDL. As reported in the same figure (left-hand side axis), also the $3 \mathrm{~dB}$ bandwidth-delay product increases with $\mathrm{K}$ for both structures (points are missing where the $3 \mathrm{~dB}$ transmission bandwidth cannot be calculated). The 2MZDL has a $3 \mathrm{~dB}$ bandwidth-delay product that is always larger than the single-stage case (up to almost two times), even when the latter has a larger $3 \mathrm{~dB}$ transmission bandwidth (e.g. $\mathrm{K}=0.14$ and $\mathrm{K}=0.86$ ).

The performance of the proposed delay lines is also evaluated in terms of group-delay bandwidth-delay product, shown in Fig. 4 (b) as function of the coupling coefficient $\mathrm{K}$ (black squares for the 1MZDL and blue circles for the 2MZDL). The use of the group-delay bandwidth allows to compare the performance also with a lossless all-pass ring-resonator delay line that has an infinite $3 \mathrm{~dB}$ transmission bandwidth [6], [8]. We consider here a single-stage ring equipped with a tunable coupler both at the resonant frequency and off-resonance (green dashed and brown dot-dashed line, respectively, in Fig. 4 (b)). Also for this structure the normalized group delay
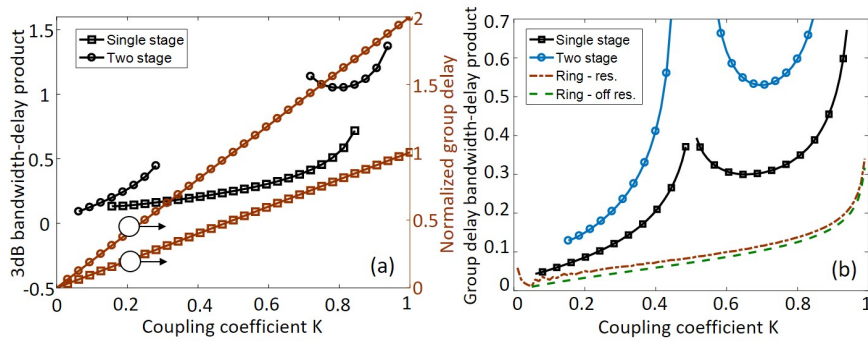

Fig. 4. (a) $3 \mathrm{~dB}$ bandwidth-delay product (left-hand side axis) and normalized group delay (right-hand side axis) as function of coupling coefficient $\mathrm{K}$ for 1MZDL and 2MZDL. (b) Group-delay bandwidth-delay product for 1MZDL and $2 \mathrm{MZDL}$ and a single-stage ring-resonator delay line used both at the resonant frequency and off-resonance. as function of frequency and coupling coefficient $\mathrm{K}$ can be analytically calculated [9].

As can be seen, the 2MZDL has a bandwidth-delay product that is always larger than the single-stage structure (as in the case of the $3 \mathrm{~dB}$ transmission bandwidth) even if the the 1MZDL has a larger group-delay bandwidth (see Fig. 3(b)). For both structures the product increases with $\mathrm{K}$ and goes to infinite for $\mathrm{K}=0, \mathrm{~K}=0.5$ and $\mathrm{K}=1$ because the group-delay bandwidth is not defined. Both Mach-Zehnder-based delay lines have a group-delay bandwidth-delay product larger that the ring-based delay line. For the ring, the product is almost identical in the resonance and off-resonance cases because the much larger group delay obtained at the resonant frequency is compensated by a corresponding much narrower group-delay bandwidth. In case of $2 \mathrm{MZDL}$, for values of $\mathrm{K}$ greater than 0.4 , the product has increased by factor of about 2 compared to $1 \mathrm{MZDL}$ and by factor of about 5 compared to the ring resonator.

\section{EXPERIMENTAL CHARACTERIZATION}

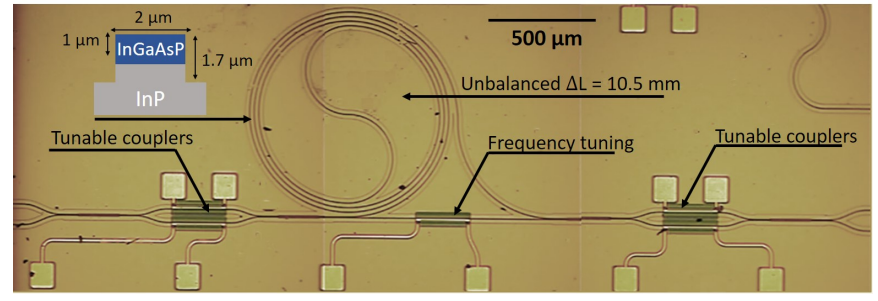

Fig. 5. Photograph of the realized Mach-Zehnder-based delay line with two tunable couplers.

In this section, we present the experimental results of a 1MZDL, the 2MZDL being under fabrication and not yet available. Figure 5 shows a top-view optical photograph of the delay line fabricated through a JePPIX Multi-Pwoifct Wafer Run on an InP-based technological platform [1 15]. The optical rib-shaped waveguide is $2 \mu \mathrm{m}$ wide with a $1 \mu \mathrm{m}$-thick InGaAsP layer (core) and etch depth of $1.7 \mu \mathrm{m}$ (see inset of Fig. 5). The estimated propagation losses are about $2 \mathrm{~dB} / \mathrm{cm}$ and the minimum bending radius is $150 \mu \mathrm{m}$. Balanced $2 \times 2$ Mach-Zehnder interferometers are used as tunable couplers, realized with multi-mode interference couplers and $250-\mu \mathrm{m}-$ long thermo-optic phase shifters $\left(\mathrm{V}_{\pi}=4.8 \mathrm{~V}\right)$. A further thermo-optic phase shifter is integrated in the shorter arm of the unbalanced Mach-Zehnder to tune the operative frequency of the delay line. The unbalance length $\Delta \mathrm{L}$ is $10.5 \mathrm{~mm}$, providing a maximum group delay variation for the TE polarized mode of about $124 \mathrm{ps}$, being $\mathrm{n}_{g}=3.54$. The reported device occupies an area of about $4 \times 1.3 \mathrm{~mm}^{2}$. This figure could be easily reduced by a factor of $\Omega$ avoiding the use of the spiral. As a comparison, a race-track ring resonator realized with the same waveguide and used gut of resonand Dould require a footprint of about $5 \times 5 \mathrm{~nm}^{2}$ [16]. The spectral characterization was performed in the optical domain using an Optical Vector Analyser that allows to characterize both power and group delay spectra. 

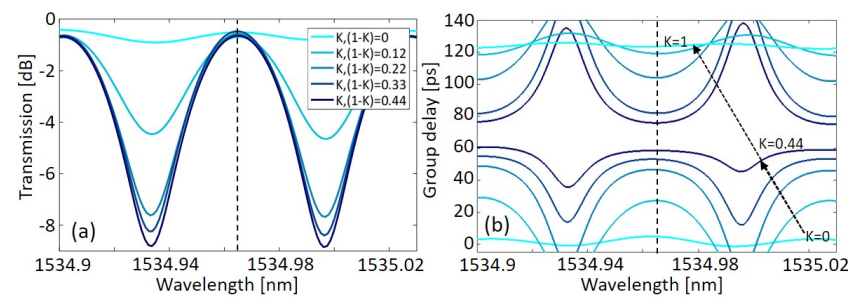

Fig. 6. Measured (a) normalized transmission and (b) group delay spectra of the 1MZDL for different values of $\mathrm{K}$. The unbalance length is $\Delta \mathrm{L}=10.5$ $\mathrm{mm}$.

The measured normalized spectral power transmission and group delay are shown in Fig. 6 (a) and (b) for several coupling coefficient $\mathrm{K}$ (TE-polarized mode) in the wavelength range between $\lambda=1534.9 \mathrm{~nm}$ and $\lambda=1535.03 \mathrm{~nm}$. The vertical dashed lines mark the operative wavelength $\lambda_{o}=$ $1534.96 \mathrm{~nm}$. The coupling coefficient of two tunable couplers are tuned by feeding a suitable current to the phase shifters. The phase shifter integrated in the shorter arm of the MachZehnder was used to compensate for the effects of thermal cross-talk and the chip was mounted on a holder with a feedback thermocontroller to stabilize the overall temperature. The measured spectral characteristics are in good agreement with the simulations shown in Fig. 2(a) and (b). For $\mathrm{K} \simeq 0$ and $\mathrm{K} \simeq 1$, the transmission and group delay spectra are almost wavelength independent and the latter is minimum at $\mathrm{K} \simeq 0$ and maximum at $\mathrm{K} \simeq 1$ (light blue lines). By tuning the two coupling coefficients $\mathrm{K}$, at the central wavelength the delay is varied while the normalized power transmission remains almost constant (except for the additional propagation losses, smaller than $2.5 \mathrm{~dB}$, induced by the propagation in the longer Mach-Zehnder arm, not reported in the figure). For values of $\mathrm{K}$ close to 0.5 the Mach-Zehnder response is formed and deep notches appear in the transmission spectrum. The measured $3 \mathrm{~dB}$ bandwidth around the operative wavelength is always larger than $4.5 \mathrm{GHz}$.

Figure 7 reports the measured $3 \mathrm{~dB}$ bandwidth-delay product (left-hand side axis) and group delay dependence on coupling coefficient $\mathrm{K}$ at the operative wavelength (right-hand side axis). The group delay (orange stars) has a linear dependence on $\mathrm{K}$ with a measured tuning range of about 124 ps, corresponding to a measured FSR of $8 \mathrm{GHz}$ (see Fig. 6(a)), in accordance with the designed value (solid orange line). The comparison of measured bandwidth-delay product (black square marker) with simulation (solid black line) is also in good agreement, the deviations mainly depending on the evaluation of the value of $\mathrm{K}$ from the current fed to the two tunable couplers. The simulated results are the same of Fig. 4(b) for a 1MZDL. As expected, the product goes to infinite for values of $\mathrm{K}$ around zero and slowly grows with $\mathrm{K}$ up to 0.7 when again goes to infinite.

\section{CONCLUSION}

We have presented an architecture based on cascaded MachZehnder interferometers implementing a non-resonant optical delay line. The group delay of the device can be continuously

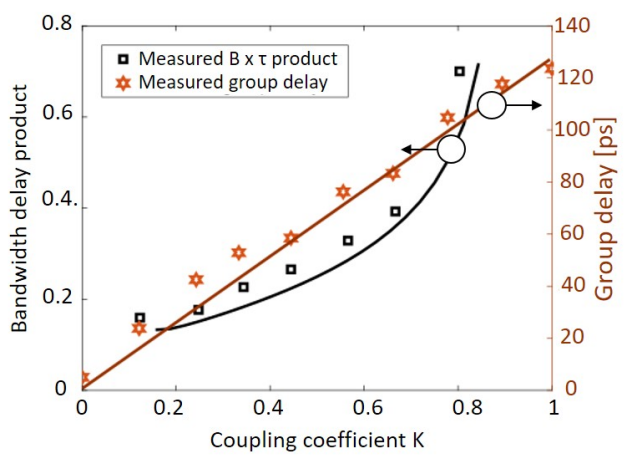

Fig. 7. 3dB bandwidth-delay product and group delay as function of the coupling coefficient obtained from measured transmission and group delay spectra. Group delay is measured at $\lambda=1534.97 \mathrm{~nm}$. Solid black and brown lines are simulation results.

tuned without affecting power transmission at the operative frequency, ensuring hitless control. The use of two cascaded stages with the described control scheme allows to double both the tunability range of the group delay and the group-delay bandwidth-delay product compared to a single-stage device. It also increases the $3 \mathrm{~dB}$ device bandwidth by $27 \%$. Compared to a ring-based delay line, the proposed architecture has a group-delay bandwidth-delay product almost five times larger. A single-stage device was experimentally demonstrated, with a group-delay tunability range of 124 ps over a $3 \mathrm{~dB}$ transmission bandwidth of $4.5 \mathrm{GHz}$.

\section{ACKNOWLEDGMENT}

The authors would like to gratefully acknowledge Erasmus Mundus LEADERS project for PhD scholarship of Abi Waqas.

\section{REFERENCES}

[1] A. Melloni et al., "Continuously tunable 1 byte delay in coupledresonator optical waveguides," Optics letters, vol. 33, no. 20, pp. 23892391, 2008.

[2] R. L. Moreira et al., "Integrated ultra-low-loss 4-bit tunable delay for broadband phased array antenna applications," IEEE Photon. Technol. Lett, vol. 25, no. 12, pp. 1165-1168, 2013.

[3] J. Capmany and D. Novak, "Microwave photonics combines two worlds," Nature photonics, vol. 1, no. 6, pp. 319-330, 2007.

[4] F. Xia et al., "Ultracompact optical buffers on a silicon chip," Nature photonics, vol. 1, no. 1, pp. 65-71, 2007.

[5] J. Cardenas et al., "Wide-bandwidth continuously tunable optical delay line using silicon microring resonators," Optics express, vol. 18 , no. 25 , pp. 26525-26534, 2010.

[6] L. Zhuang et al., "Novel low-loss waveguide delay lines using vernier ring resonators for on-chip multi- $\lambda$ microwave photonic signal processors," Laser \& photonics reviews, vol. 7, no. 6, pp. 994-1002, 2013.

[7] A. Melloni et al., "Tunable delay lines in silicon photonics: coupled resonators and photonic crystals, a comparison," IEEE Photonics Journal, vol. 2, no. 2, pp. 181-194, 2010.

[8] L. Zhuang et al., "Low-loss, high-index-contrast si 3 n 4/sio 2 optical waveguides for optical delay lines in microwave photonics signal processing," Optics express, vol. 19, no. 23, pp. 23 162-23 170, 2011.

[9] Y. Liu et al., "Ultra-low-loss silicon nitride optical beamforming network for wideband wireless applications," IEEE Journal of Selected Topics in Quantum Electronics, vol. 24, no. 4, pp. 1-10, 2018.

[10] G. Lenz et al., "Optical delay lines based on optical filters," IEEE Journal of Quantum Electronics, vol. 37, no. 4, pp. 525-532, 2001.

[11] M. S. Rasras et al., "Integrated resonance-enhanced variable optical delay lines," IEEE photonics technology letters, vol. 17, no. 4, pp. 834836, 2005. 
[12] R. Moreira et al., "Programmable eye-opener lattice filter for multichannel dispersion compensation using an integrated compact low-loss silicon nitride platform," Optics express, vol. 24 , no. 15 , pp. 16732 $16742,2016$.

[13] D. Melati et al., "Wideband integrated optical delay line based on a continuously tunable mach-zehnder interferometer,' IEEE Journal of Selected Topics in Quantum Electronics, vol. 24, no. 1, pp. 1-8, 2018.

[14] JePPIX. [Online]. Avaialable: http://www.jeppix.eu. Last accessed on: 25-06-2018

[15] D. Melati et al., "Validation of the building-block-based approach for the design of photonic integrated circuits," Journal of Lightwave Technology, vol. 30, no. 23, pp. 3610-3616, 2012.

[16] N. Tessema et al., "A tunable si 3 n 4 integrated true time delay circuit for optically-controlled k-band radio beamformer in satellite communication," Journal of Lightwave Technology, vol. 34, no. 20, pp. 4736-4743, 2016. 